\title{
Understanding the Art and Science of Physician Well Being
}

\section{A. A. Mamun Hussain ${ }^{1}$}

Physicians although indoctrinated within a culture that boasts of superhuman stamina, but often have underdeveloped skills sets for self-care and for achieving a meaningful work-life balance.

Increasing complexities, as observed in today's health care environment have introduced a number of different factors distracting physicians from their primary goal to provide the best possible care. At times the resulting levels of frustration, dissatisfaction, stress and burnout can affect their attitudes and behaviors to a point where they become disruptive and their actions can negatively impact relationship that adversely affect patient care outcomes. In our experience and from the existing literature, such conditions may start early in medical school and continue throughout various stages of professional and personal development. Physician stress and burnout, although considered a occupational hazard due to hard-driving personality traits, but researchers believe that a significant portion is due to potentially modifiable workplace factors. Physicians today are overwhelmed by multiple administrative requirements and time constraints and are being asked to take on more and more responsibilities that take them away from direct patient care.

It goes without saying that, physicians understand the importance of relaxation and recreation, adequate sleep, regular exercise and good nutrition, but has difficulty integrating such selfcare activities into their own lives. ACGME ${ }^{1}$ (Accreditation Council for Graduate Medical Students, 2017), in a report elucidated that-

"In the current health care environment, residents and faculty members are at increased risk for burnout and depression. Psychological, emotional and physical well-being are critical in the development of the competent, caring and resilient physician. Self-care is an important component of professionalism; it is also a skill that must be learned and nurtured in the context of the aspects of residency training.”

In this regards two major strategies could be recommended, viz, organizational intervention that target modifiable workplace stressors associated with burnout, and the development of self-care strategies and personal resiliency by the physicians themselves.

So considering the art and science of physician well being, we need to develop a 'Physician Health Program' emphasizing the journey through the life time of a physician, which meanwhile has got momentum globally in medical domain a few decade back. ${ }^{2}$ Hopefully such program would include the self-care, resilience and work-life balance of physician as well as. In many a centre, mindfulness-based course is offered for medical students, residents and health care professional. So far the technological resources ${ }^{3}$ are considered, one can also look for smart phone apps, fitness tracking device and different internet-based program, for self-learning.

As an after word, although the wellness, well being and health care considered as related construct but a physician's well being is measured by the overall evaluation of his lives (Job and life satisfaction) and job-related emotional experience (higher positive and lower negative affects). As such well-being of a physician is very much associated with better quality of Patient care. Finally, let it be a maxim, that caring for patients' demands caring for 
physicians in same magnitude, as well as, for improving our total doctoring process.

\section{References:}

1. ACGME, 2017. www.acgme.org/portals/O/PFA Assets/
2. Physician Health Programs: The US Model. Gary DC, Bradley H, Robert L D et al. Springer Int. Pub. AG. 2017

3. Self care, Resilience and work-life Balance. Linda LMW \& Cynthic MI. Springer Int. Pub. AC. 2017

All Correspondence to

Prof A. A. Mamun Hussain Department Of Psychiatry,

Rajshahi Medical College E-mail: mamun.hussain.bd@gmail.com 